\title{
One-Step Preparation of Graphene Oxide/Cellulose Nanofibril Hybrid Aerogel for Adsorptive Removal of Four Kinds of Antibiotics
}

\author{
Jin Wang, ${ }^{1}$ Qiufang Yao, ${ }^{1}$ Chengmin Sheng, ${ }^{1}$ Chunde Jin,, ${ }^{1,2}$ and Qingfeng Sun ${ }^{1,2}$ \\ ${ }^{1}$ School of Engineering, Zhejiang A\&F University, Linian, China \\ ${ }^{2}$ Key Laboratory of Wood Science and Technology, Linian, China \\ Correspondence should be addressed to Chunde Jin; zafujincd@163.com and Qingfeng Sun; qfsun@zafu.edu.cn
}

Received 15 November 2016; Revised 18 January 2017; Accepted 31 January 2017; Published 16 April 2017

Academic Editor: Qin $\mathrm{Hu}$

Copyright (C) 2017 Jin Wang et al. This is an open access article distributed under the Creative Commons Attribution License, which permits unrestricted use, distribution, and reproduction in any medium, provided the original work is properly cited.

\begin{abstract}
Via a one-step ultrasonication method, cellulose nanofibril/graphene oxide hybrid (GO-CNF) aerogel was successfully prepared. The as-prepared GO-CNF possessed interconnected 3D network microstructure based on GO nanosheets grown along CNF through hydrogen bonds. The aerogel exhibited superior adsorption capacity toward four kinds of antibiotics. The removal percentages $(R \%)$ of these antibiotics were $81.5 \%, 79.5 \%, 79.1 \%$, and $73.9 \%$ for Doxycycline (DXC), Chlortetracycline (CTC), Oxytetracycline (OTC), and tetracycline (TC), respectively. Simultaneously, the adsorption isotherms were well fitted to Langmuir model and kinetics study implied that the adsorption process was attributed to pseudo-second-order model. The maximum theoretical adsorption capacities of GO-CNF were 469.7, 396.5, 386.5, and $343.8 \mathrm{mg}^{-1}$ for DXC, CTC, OTC, and TC, respectively, calculated by the Langmuir isotherm models. After five cycles, importantly, the regenerated aerogels still could be used with little degradation of adsorption property. Consequently, the as-synthesized GO-CNF was a successful application of effective removal of antibiotics.
\end{abstract}

\section{Introduction}

With desirable antimicrobial activity, high quality, and low cost, tetracyclines are of widespread use of human therapy and livestock industry [1]. Unfortunately, tetracyclines have been regularly detected in surface water, groundwater, and even drinking water, resulting in harmfulness to environment and our human health $[2,3]$. Therefore, it has been urgent that effective measures should be taken to remove tetracyclines from contaminative water. Recently, a variety of methods have been explored to decontaminate antibiotic such as adsorption [4], photocatalytic degradation [5], catalytic degradation [6], advanced oxidation [7, 8], and biodegradation [9]. Among these various methods, adsorption is a superior and widely used method owing to its accessibility, being environmentally benign, and high efficiency [10]. In fact, a number of (chemical or physical) sorbent materials, including various kinds of adsorbents, including $\mathrm{HCl}$ modified zeolite [11], alkali biochar [12], anaerobic granular sludge [13], activated carbons [14, 15], multiwalled carbon nanotubes [16,17], bamboo charcoal [18], and graphene oxide [19], have been applied for eliminating antibiotics from aqueous solutions. However, having suffered from low removal capacities, difficult separation, secondary environmental pollution, and unsatisfactory recycling ability, these adsorbents have hampered greatly practical applications. Therefore, it is an urgent demand to develop new adsorbents with reusability and efficiency to remove antibiotics in water.

As porous nanostructured materials, aerogels have been applied in considerable fields due to their superior properties like low density, high specific surface area, and excellent adsorption [20]. Specifically, aerogels are based on cellulose nanofibers (CNF) networks with outstanding physical properties such as high surface-to-volume area and high aspect ratio; they show an attractive flexibility, ductility, hierarchically porous structure, and many other excellent features [21]. Therefore, materials based on CNF aerogels with 3D structures are the current focus of a tremendous 
TABLE 1: The molecular structures of tetracyclines and their abbreviations.

(n)

surge of interest in adsorption applications. On the other hand, with extremely hydrophilicity, ultrahigh theoretical surface area and abundant surface oxygen containing groups, graphene oxide (GO) nanosheets have been applied to field for adsorbing pollutants such as antibiotics [22], dyes (methylene blue, Basic Red 12, and triphenylmethane), and heavy metal ions (copper, zinc, cadmium, and lead) [23, 24] from water. However, there exists certain defect in the use of conventional powder GO-base materials, such as segregating after the reaction. Hence, materials with ease of separation operation as well as good hydrophilic properties are urgently needed in antibiotics removal application.

In this paper, GO-CNF with ease of separation operation as well as good hydrophilic properties was prepared by using one-step ultrasonication method. The results demonstrated the significance of the absorption properties within the GO$\mathrm{CNF}$ for application in removal of antibiotics. Moreover, the GO-CNF could be regenerated several times using an alkali washing procedure with little loss in multiple antibiotics removal performance.

\section{Materials and Methods}

2.1. Materials. Four kinds of tetracyclines were shown in Table 1 and were supplied by Aladdin Industrial Co., Ltd. The moso bamboo (Phyllostachys heterocycla) was obtained from Zhejiang Province in China. All other chemicals were analytical grade and used as received.

\subsection{Preparation of Cellulose Nanofibril/Graphene Oxide Hybrid} (GO-CNF) Aerogel by One-Step Ultrasonication Method. Graphene oxide (GO) and pure cellulose were prepared according to the literatures methodologies $[25,26]$, respectively. In a typical process, pure cellulose $(80 \mathrm{mg})$ and $\mathrm{GO}(100 \mathrm{mg})$ were put in distilled water $(100 \mathrm{~mL})$ under vigorous ultrasonication for $30 \mathrm{~min}$. Ultrasonic treatment was performed at $60 \mathrm{kHz}$ with a $25 \mathrm{~mm}$ diameter titanium horn under a duty cycle with an outpower of $1000 \mathrm{~W}$. During ultrasonication, the samples were kept in an ice bath to avoid overheating. Afterward, the specimens were freeze-dried using a lyophilizer (Scientz-10N, Ningbo Scientz Biotechnology Co., Ltd., China) for 48 h.

2.3. Characterizations. The morphologies of the aerogels were characterized by scanning electron microscopy (SEM, FEI, Quanta 200, USA) and transmission electron microscope (TEM, FEI, Tecnai G20, USA). Atomic force microscopic (AFM) images were taken on a MultiMode Nanoscope III scanning probe microscopy (SPM) system (Veeco, USA). Crystalline structures were identified by X-ray diffraction technique (XRD, Rigaku, D/MAX 2200, Japan), operating with $\mathrm{Cu} \mathrm{K} \alpha$ radiation $(\lambda=1.5418 \AA)$ at a scan rate $(2 \theta)$ of $2^{\circ} \mathrm{min}^{-1}$ and the accelerating voltage of $40 \mathrm{kV}$ and the applied current of $30 \mathrm{~mA}$. The surface elemental composition analyses were conducted based on the X-ray photoelectron spectroscopy (XPS, Thermo Fisher Scientific-K-Alpha 1063, $\mathrm{UK}$ ) with an $\mathrm{Al} \mathrm{Ka}$ monochromatic X-ray source, in which all of the binding energies were calibrated with reference to the $\mathrm{C}$ 1s peak $(284.8 \mathrm{eV})$. The Brunauer-Emmet-Teller (BET) surface area $\left(S_{\mathrm{BET}}\right)$ and pore properties of the aerogels were determined from $\mathrm{N}_{2}$ adsorption-desorption experiments at $-196^{\circ} \mathrm{C}$ using an accelerated surface area and porosimetry system (ASAP 2020, Micromeritics instrument Ltd., USA). Meanwhile, the pore volume and pore size distribution were estimated by the Barrett-Joyner-Halenda (BJH) method.

\subsection{The Adsorption Experiments}

2.4.1. The Adsorption of Antibiotics Experiment. All batch experiments of the antibiotics adsorption were carried out in 
$50 \mathrm{~mL}$ Erlenmeyer flask at a temperature controlled with water bath shaker at $160 \mathrm{rpm}$, each containing $8 \mathrm{mg}$ GO$\mathrm{CNF}$ and $40 \mathrm{~mL}$ of antibiotics solution. The mixed GOCNF solutions were incubated with antibiotics overnight at $25^{\circ} \mathrm{C}$ and covered by aluminum foil to refrain probable photodegradation of antibiotics. The final supernatant was regarded as the residual concentration of antibiotics and determined by a UV-vis spectrophotometer (ERSEE TU1900 , China). The removal percentage $(R \%)$ and the amount of antibiotics absorbed $\left(q_{e}, \mathrm{mg} \cdot \mathrm{g}^{-1}\right)$ were calculated using (1) and (2), respectively:

$$
\begin{aligned}
R \% & =\left[\frac{C_{o}-C_{e}}{C_{o}}\right] * 100 \%, \\
q_{e} & =\left[\frac{C_{o}-C_{e}}{m}\right] * V,
\end{aligned}
$$

where $C_{o}\left(\mathrm{mg} \cdot \mathrm{L}^{-1}\right)$ is the initial concentration of the antibiotics, $C_{e}\left(\mathrm{mg} \cdot \mathrm{L}^{-1}\right)$ is the equilibrium concentration in solution of the antibiotics, $V(\mathrm{~L})$ is the volume of solution, and $m$ (g) is the weight of the GO-CNF.

\subsubsection{Adsorption Kinetics Experiments and Adsorption Isotherms} Experiments. GO-CNF was added to antibiotic solutions $(40 \mathrm{~mL})$ with the aerogel: antibiotic aqueous solution ratio of $1 \mathrm{mg}$ : $5 \mathrm{~mL}$. Specimens were shaken under agitation speed of $160 \mathrm{rpm}$ at $25^{\circ} \mathrm{C}$ for different times; the supernatants were separated from the solid phase and directly analyzed by UV-vis spectrophotometer. Adsorption kinetics were conducted in triplicate. Antibiotic adsorption isotherms of GO-CNF were performed on in batches just like kinetics experiments instead of different amount of adsorbent. In a separate adsorption experiment, the exhausted aerogel suspension with the remaining antibiotic solution after the last adsorption cycle was used to test recycle of adsorbent as described below.

2.4.3. The Recyclability Experiments of the GO-CNF. The adsorbed GO-CNF was immerged into 5 wt.\% $\mathrm{NaOH}$ solution for $5 \mathrm{~h}$. After desorption, the recovered aerogels were separated and washed by distilled water. Afterward, the final sample was freeze-dried under vacuum for the next run of adsorption tests. The regenerated GO-CNF was reused for five repeated cycles following the above steps.

\section{Results and Discussion}

Figure 1 depicted the typical SEM and AFM images of CNF and GO-CNF, respectively. Both of aerogels exhibited an interconnected porous structure with pore sizes ranging from several hundreds of nanometers to a few micrometers. Compared with fibrous structure of CNF (Figure $1(\mathrm{a})$ ), the microstructure of GO-CNF exhibited a hierarchical structure with interconnected 2D sheetlike networks (Figures 1(b) and 1(c)), which exhibited an interconnected spongelike porous structure with pore sizes ranging from several hundreds of nanometers to a few micrometers and this could be proved in the data of pore size distribution and specific area in Figure 3. For GO-CNF (Figure 1(f)), the pores were obvious and more uniform and had smaller diameters compared to those of CNF. This might be attributed to the hydrogen bonding between the CNF chains and the formation of a 3D network structure which does not collapse upon sublimation. Further learned from AFM images of the specimens, the CNF which presented individual flexible fibril was roughly $65 \mathrm{~nm}$ in diameter (Figure 1(d)). For GOCNF, GO existing in the sheet-like shapes was supported by a skeleton of CNF to form an interconnected network sheets (Figure 1(e)). The thickness, measured from the AFM image, was about $4.7 \mathrm{~nm}$, which was consistent with the data reported in the literature, indicating the formation of multilayered GO.

Figure 2 presented XRD patterns and XPS spectra of the $\mathrm{CNF}$ and the GO-CNF. In Figure 2(a), the diffraction peaks at about $22^{\circ}$ and $16^{\circ}$ were attributed to the typical reflection planes (002) and (101) of cellulose I based on the JCPDS data (03-0289) [27]. As for GO-CNF, differently, an intense peak at $2 \theta=10.3^{\circ}$ could be observed, corresponding to the (001) diffraction planes of GO [28].

The surface chemistry of CNF and GO-CNF was characterized by X-ray photoelectron spectroscopy (XPS). The fully scanned spectra in Figure 2(b) illustrated that both the $\mathrm{O}$ and $\mathrm{C}$ elements existed simultaneously of the $\mathrm{CNF}$ and the GO$\mathrm{CNF}$. The variation of $\mathrm{C} / \mathrm{O}$ ratio revealed the changes in the elemental composition of the specimens. Compared with the $\mathrm{C} / \mathrm{O}$ ratio of 1.42 in the $\mathrm{CNF}$, the $\mathrm{C} / \mathrm{O}$ ratio for the $\mathrm{GO}-\mathrm{CNF}$ was 1.53 , further indicating the highly efficient conjugation during the one-step ultrasonication treatment.

Figure 2(c) exhibited the high resolution XPS C1s spectra of specimens. The curves were fitted considering the following contributions: $\mathrm{O}-\mathrm{C}=\mathrm{O}(289.8 \mathrm{eV}), \mathrm{C}=\mathrm{O}(287.8 \mathrm{eV})$, $\mathrm{C}-\mathrm{O}(286.5 \mathrm{eV}), \mathrm{C}=\mathrm{C}$ or $\mathrm{C}-\mathrm{C}$, or $\mathrm{C}-\mathrm{H}(284.4 \mathrm{eV})$ [29]. The $\mathrm{C}=\mathrm{C} / \mathrm{C}-\mathrm{C}$ peak intensity of $\mathrm{GO}-\mathrm{CNF}$ increased obviously compared with $\mathrm{CNF}$, indicating that GO-CNF was consisted of GO and CNF. In addition, there was still a large number of oxygen containing functional groups, which mainly are derived from CNF in the GO-CNF. The other peaks at 287.8 and $289.1 \mathrm{eV}$ were attributed to $\mathrm{C}=\mathrm{O}$ bonds and $\mathrm{O}-\mathrm{C}=\mathrm{O}$ bonds, respectively, which were typical functional groups in GO. In conclusion, GO were intimately combined with CNF through hydrogen bonds.

Figure 2(d) showed the high resolution XPS spectra of O1s of specimen. XPS energy spectrum of O1s could be fitted considering the following contributions: $\mathrm{C}-\mathrm{OH}$ $(533.0 \mathrm{eV})$ and $\mathrm{C}-\mathrm{OH} \cdots \mathrm{O}(532.8 \mathrm{eV})$. Compared to CNF, the $\mathrm{C}-\mathrm{OH}$... O peak intensity of GO-CNF increased obviously, indicating that GO were effectively combined with CNF through hydrogen bonds. In addition, there was a new peak of at $531.4 \mathrm{eV}$, which could be assigned to $\mathrm{O}=\mathrm{C}$ derived from GO in GO-CNF.

Figure 3 presented the $\mathrm{N}_{2}$ adsorption-desorption isotherms and pore diameter distributions of the aerogels. The absorption isotherms of both the CNF and GO/CNF aerogel (Figure 3(a)) were attributed to type-IV adsorption isotherm, according to the IUPAC classification. GO-CNF was greatly 


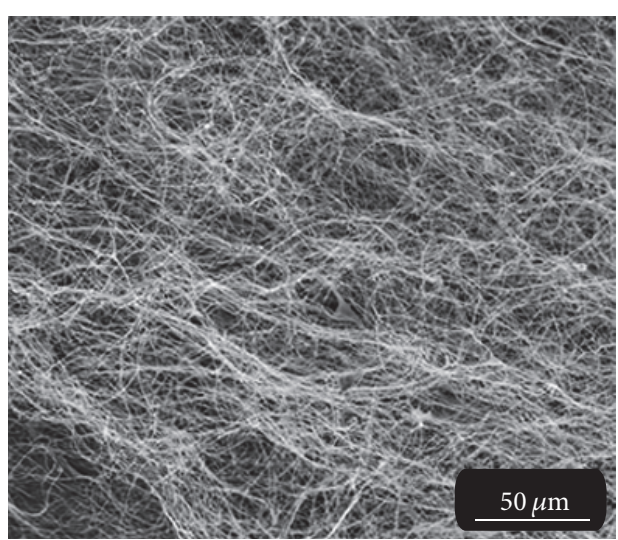

(a)

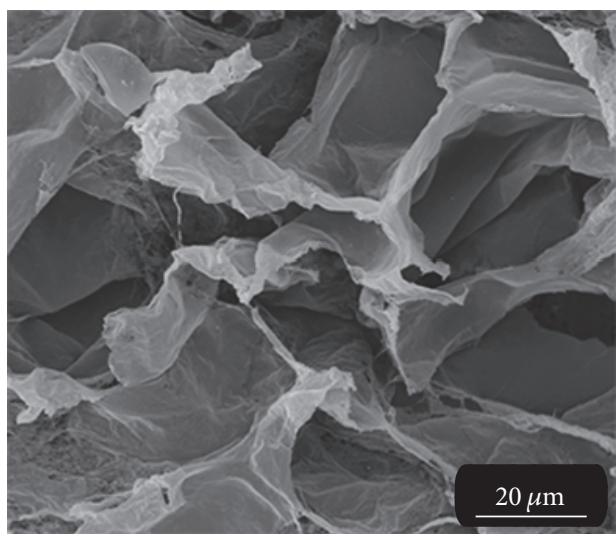

(c)

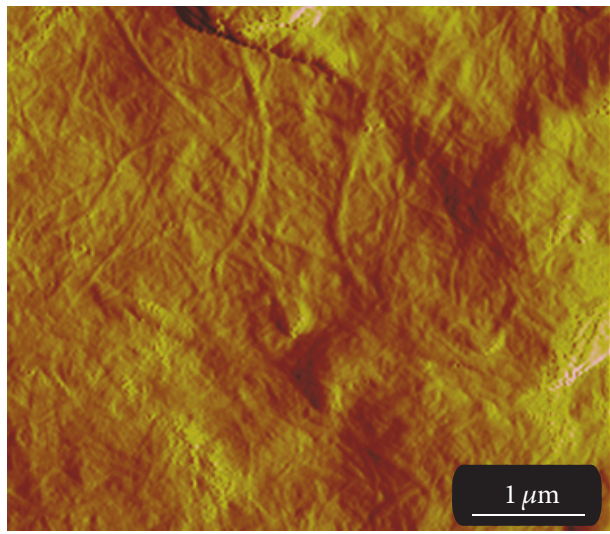

(e)

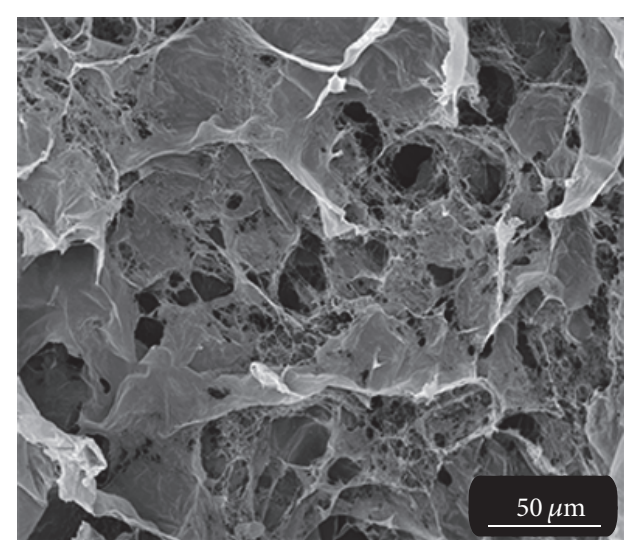

(b)

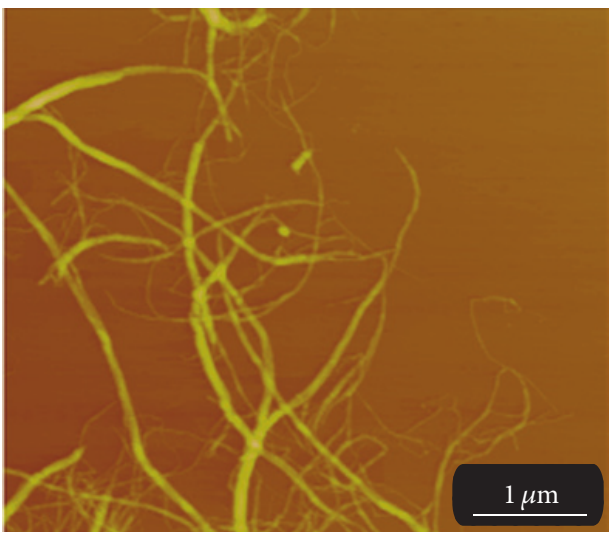

(d)

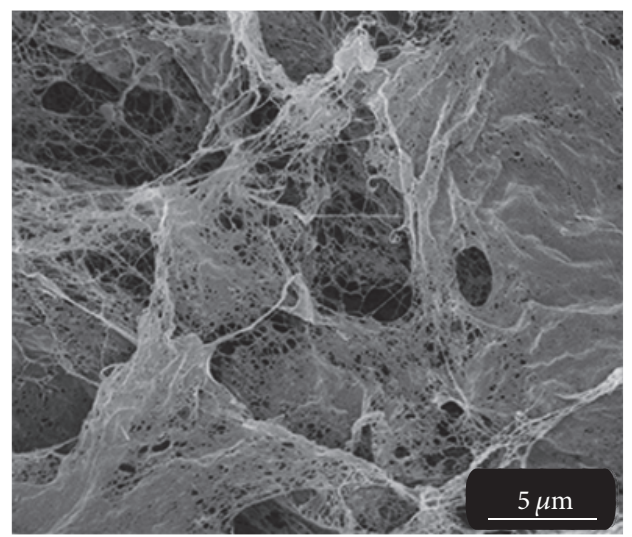

(f)

FIGURE 1: SEM images and AFM images of the CNF (a and d) and GO-CNF (b, c, f, and e), respectively.

increased in terms of $S_{\mathrm{BET}}$ and pore volume compared with that of CNF. In detail, $S_{\mathrm{BET}}$ and pore volume of GO-CNF were $89.9 \mathrm{~m}^{2} \cdot \mathrm{g}^{-1}$ and $0.4 \mathrm{~cm}^{3} \cdot \mathrm{g}^{-1}$, respectively, while $\mathrm{CNF}$ were as low as $4.8 \mathrm{~m}^{2} \cdot \mathrm{g}^{-1}$ and $0.05 \mathrm{~cm}^{3} \cdot \mathrm{g}^{-1}$, respectively. Figure 3(b) exhibited growth of mesoporous structure in GOCNF instead of CNF. These results indicated GO-CNF with ultrahigh surface areas and adsorbing site might be effective in removal of antibiotics.

Figure 4 showed synthesis of GO-CNF via one-step ultrasonication method. During the ultrasonic treatment, one hand, CNF were formed springing from the pure cellulose by cavitation effect [30]. In detail, $80 \mathrm{mg}$ of the purified cellulose suspension was passed through an ultrasonic processor for nanofibrillation, which is based on an ultrasonication mechanism: ultrasonication causes the natural fibres to disassemble into nanofibers in water via cavitation, formation, growth, and implosive collapse of bubbles in the solution. During ultrasound, for example, small gas bubbles (cavities) will be generated in a cellulose aqueous suspension. Such small gas bubbles are able to absorb energy from the soundwaves and 


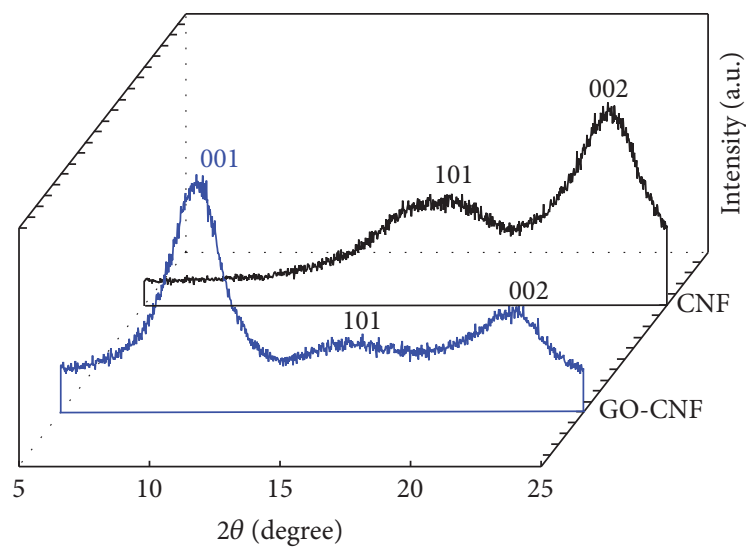

(a)

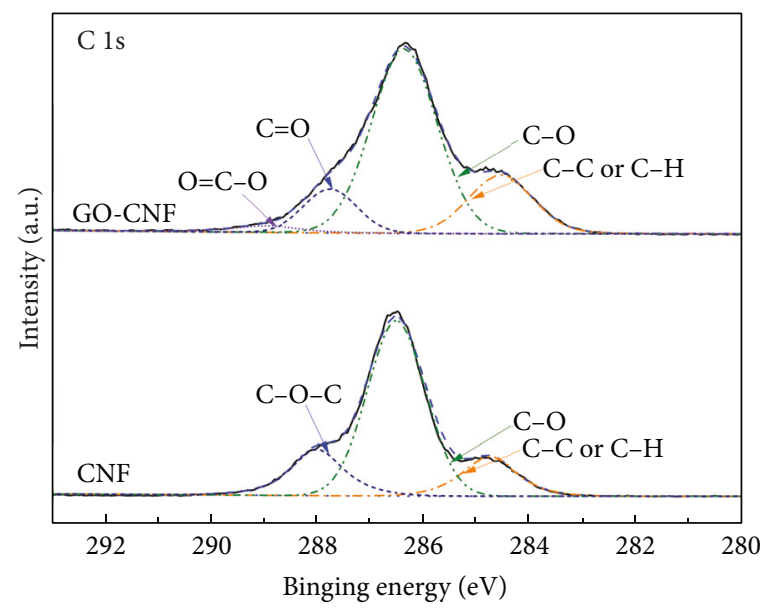

(c)

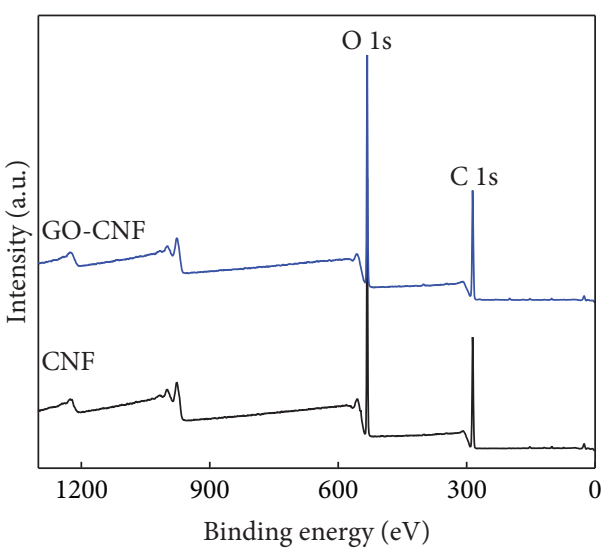

(b)

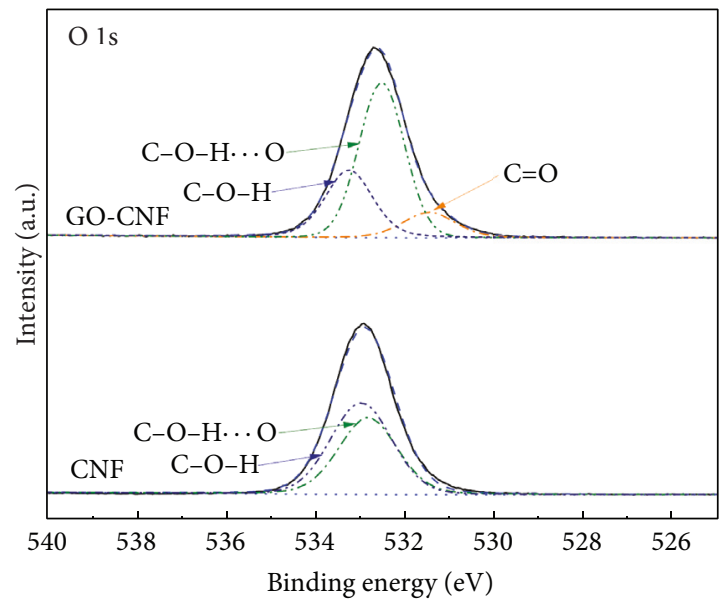

(d)

FigURE 2: (a) XRD patterns, (b) survey scan, (c) Cls, and (d) Ols XPS spectra of CNF and GO-CNF.

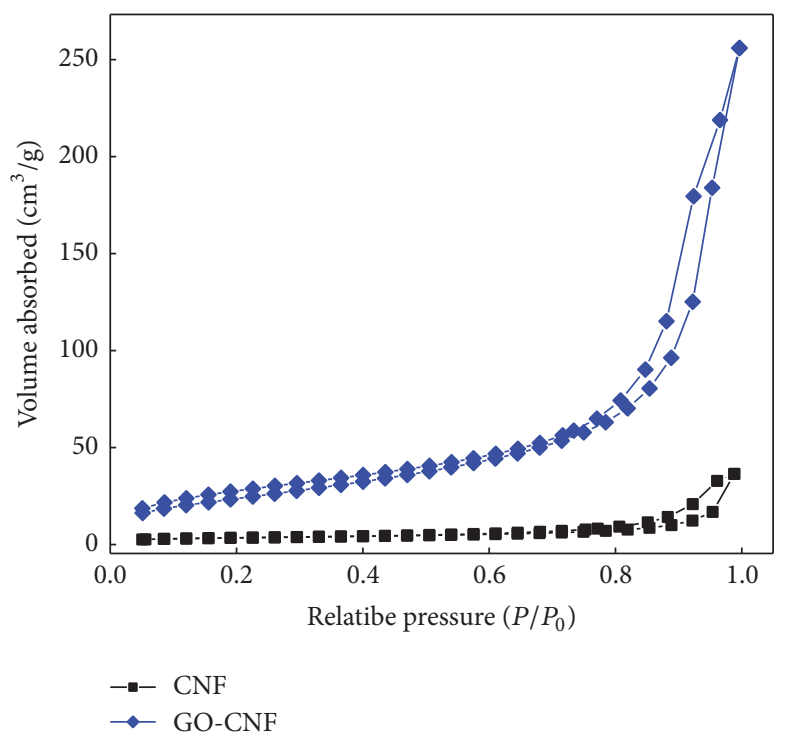

(a)

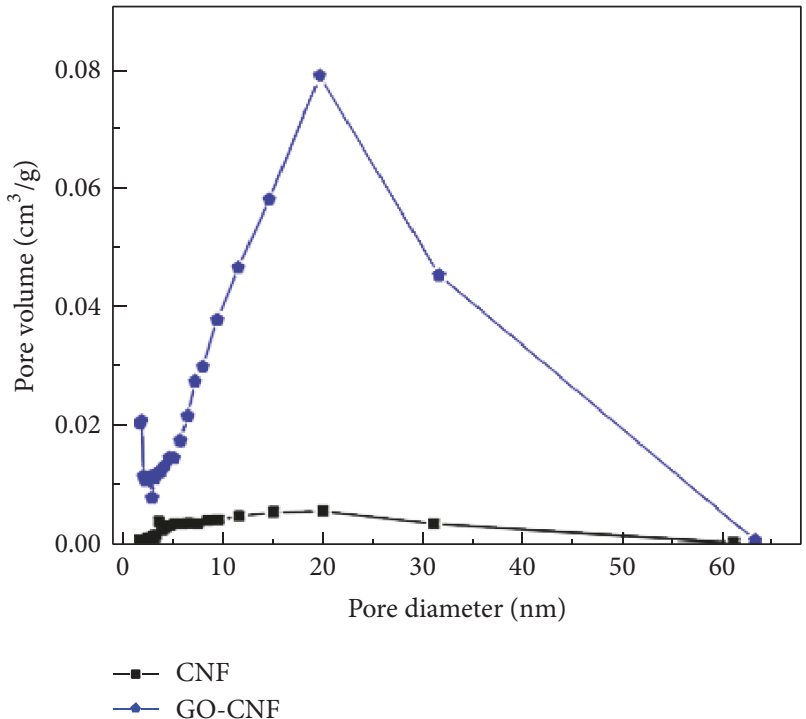

(b)

FIGURE 3: Nitrogen adsorption-desorption isotherms (a) and BJH desorption pore size distribution (b) of CNF and GO-CNF, respectively. 


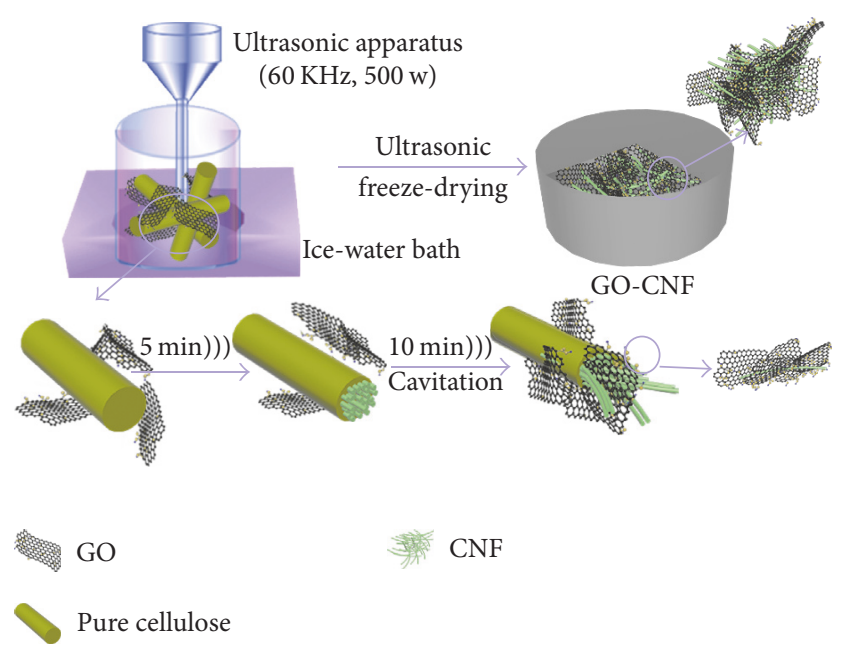

FIGURE 4: Schematic showing the synthesis of GO-CNF via one-step ultrasonication method.

TABLE 2: Kinetic properties of antibiotics removal on GO-CNF by pseudo-second-order model.

\begin{tabular}{lccc}
\hline & \multicolumn{3}{c}{ Pseudo-second-order } \\
$h\left(\mathrm{mg} \cdot(\mathrm{g} \cdot \mathrm{min})^{-1}\right)$ & $r^{2}$ \\
\hline DXC & 0.1299 & 543.6589 & 0.994 \\
CTC & 0.1286 & 684.6815 & 0.993 \\
OTC & 0.1158 & 702.6432 & 0.994 \\
TC & 0.0995 & 714.2857 & 0.992 \\
\hline
\end{tabular}

grow rapidly under high ultrasound intensities. However, the cavity implodes when it has overgrown, and the surrounding liquid rushes in. The implosion of the cavity creates an unusual environment and introduces high pressure and shock waves within a short time. This violent collapse causes direct particle-shock wave interactions and is the primary pathway to split cellulose fibres along the axial direction. Thus, the sonification impact breaks the relatively weak cellulose interfibrillar hydrogen bonding and the Van der Waals force, gradually disintegrating the microscale cellulose fibres into nanofibers. On the other hand, GO grew and aggregated to transformed lager flakes based on a skeleton of CNF, which was because GO with plentiful oxygen could interact with the hydroxyl groups and oxygen atoms in CNF through hydrogen bonds. Afterwards, during the freezedrying process, nucleation and growth of large ice crystals can occur within the network that pushed out the GOCNF from its original location [31]. Subsequent sublimation of these large crystals led to the formation of micrometersized pores in the aerogel. Moreover, GO with hydrophilic patterns (CNF) expected preferential bubble nucleation on the GO areas whose architecture ensured high availability of the adsorption sites for removal of antibiotics.

Figure 5 showed the removal percentage $(R \%)$ of antibiotics. Four kinds of antibiotics could be inordinately absorbed

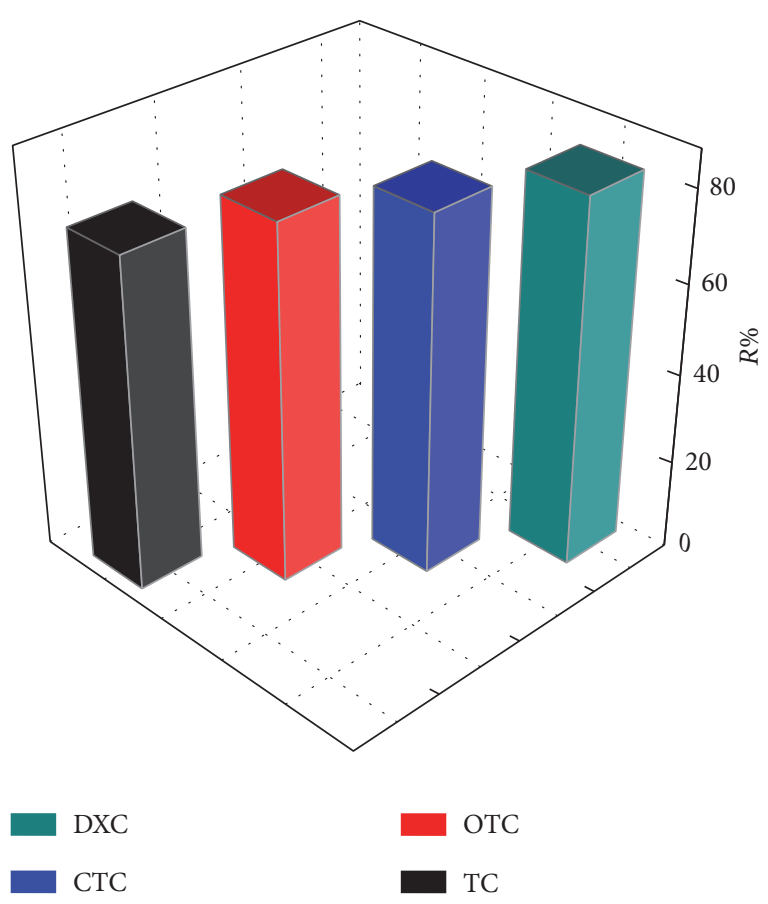

Figure 5: The $R \%$ of the antibiotics $(20 \mathrm{mg} / \mathrm{L})$ on GO-CNF $(20 \mathrm{mg} / \mathrm{L})$; temp. $25 \pm 0.1$.

on the GO-CNF. The valves of $R \%$ ranged from 73.9 to $81.5 \%$. In detail, the sequence of absorption efficiency was as follows: DXC $(81.5 \%)>$ CTC $(79.5 \%)>$ OTC $(79.1 \%)>$ TC (73.9\%), which was attributed to the different groups on the branched chain of their chemical construction. Hence, these results exhibited that more conjugated structures and hydroxyl groups could be a benefit of antibiotics adsorption.

In order to investigate the adsorption process of antibiotic by GO-CNF, the pseudo-second-order kinetics models calculated by (3) were represented as follows [32]:

$$
\begin{aligned}
\frac{t}{q_{t}} & =\frac{1}{k_{2} q_{2}^{2}}+\frac{t}{q_{2}}, \\
h & =k_{2} q_{2}^{2},
\end{aligned}
$$

where $q_{t}\left(\mathrm{mg} \mathrm{g}^{-1}\right)$ is the amount of antibiotic adsorbed on the surface of the adsorbent at any time and $k_{2}\left(\mathrm{~g} \cdot(\mathrm{mg} \cdot \mathrm{h})^{-1}\right)$ is the rate constant of adsorption in pseudo-second-order model, respectively; $q_{2}\left(\mathrm{mg} \mathrm{g}^{-1}\right)$ is the amount of antibiotic adsorbed at equilibrium.

The pseudo-second-order kinetics model described relied on the assumption that adsorption was a chemical reaction, that is, chemisorption. The obtained kinetics parameters of pseudo-second-order kinetics model for adsorption of antibiotics on GO-CNF were listed in Table 2. The pseudosecond-order model $\left(R^{2}>0.99\right)$ fitted the adsorption data well, which illustrated that there was chemical adsorption in quick adsorption of antibiotics on the GO-CNF. Moreover, the calculated adsorption capacity $\left(q_{e}\right)$ values estimated by 
TABLE 3: Langmuir and Freundlich adsorption isotherms fitting parameters of antibiotics.

\begin{tabular}{lcccccc}
\hline & & Langmuir & & \multicolumn{3}{c}{ Freundlich } \\
& $q_{m}\left(\mathrm{mg} \cdot \mathrm{g}^{-1}\right)$ & $K_{L}\left(\mathrm{~L} \cdot \mathrm{mg}^{-1}\right)$ & $r^{2}$ & $K_{F}\left(\mathrm{~L} \cdot \mathrm{mg}^{-1}\right)$ & $n$ & $r^{2}$ \\
\hline DXC & 469.74 & 0.126 & 0.997 & 49.563 & 1.687 & 0.938 \\
CTC & 396.49 & 0.117 & 0.995 & 48.847 & 1.987 & 0.964 \\
OTC & 386.53 & 0.098 & 0.996 & 46.985 & 2.145 & 0.959 \\
TC & 343.84 & 0.086 & 0.998 & 43.572 & 2.257 & 0.957 \\
\hline
\end{tabular}

the pseudo-second-order model were $96.3,89.7,87.9$, and $84.8 \mathrm{mg} \cdot \mathrm{g}^{-1}$ for DXC, CTC, OTC, and TC, respectively, which showed excellent consistency with the detected val-

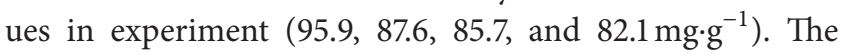
rate constant of sorption $(k)$ was $0.129,0.128,0.116$, and $0.09 \mathrm{~g} \cdot(\mathrm{mg} \cdot \mathrm{h})^{-1}$ for DXC, CTC, OTC, and TC, respectively.

As two classic adsorption models, the Langmuir and Freundlich models were applied to describe the adsorption equilibrium. The mathematical representations of the Langmuir Equation and Freundlich Equation models [33] were given as follows:

$$
\begin{aligned}
\frac{1}{q_{e}} & =\frac{1}{q_{m}}+\frac{1}{K_{L} q_{e} C_{e}}, \\
\ln q_{e} & =\ln K_{F}+\frac{1}{n} \ln C_{e},
\end{aligned}
$$

where $q_{m}\left(\mathrm{mg}^{-1} \mathrm{~g}^{-1}\right)$ is the theoretical maximum adsorption capacity per unit weight of the adsorbent and $n$ is the Freundlich linearity index. $K_{L}$ and $K_{F}$ are adsorption constants of Langmuir and Freundlich models, respectively. As an ideal model, Langmuir model was applied widely in perfect adsorbent surface and monolayer molecule adsorption. On the other hand, as an empirical model, Freundlich model was applied in the field of chemistry. The results of fitting parameters by these models were listed in Table 3. For the four kinds of antibiotics, the Langmuir $\left(R^{2}>0.99\right)$ fitted the adsorption data well and the Freundlich model $\left(R^{2}>\right.$ 0.93 ) fitted reasonably. Hence, at certain concentration levels instead of high one, the Langmuir model globally fitted well, which exhibited the limitation of the hypothesis about a monolayer adsorption. Moreover, Freundlich model would be more fitted at high concentration which exhibited that it was chemistry adsorption progress. Simultaneously, from Langmuir model, the ideal maximum adsorption capacity $\left(q_{m}\right)$, a model fitting parameter, was determined to be 469.7 , 396.5, 386.5, and $343.8 \mathrm{mg}^{-1}$ for DXC, CTC, OTC, and TC, respectively. Using the Freundlich model, the obtained Freundlich constant $\left(K_{F}\right.$ was $49.6,48.8,46.9$, and $43.6 \mathrm{~L} \cdot \mathrm{mg}^{-1}$ for DXC, CTC, OTC, and TC, resp.) was higher than what was reported by Ghadim et al. [22].

The possible adsorption mechanisms of the antibiotics by GO-CNF were based on the following properties in Figure 6 .
Firstly, with primarily $\pi-\pi$ stacking, GO-CNF could act as electron acceptors and be advantageous for adsorbing the antibiotics by unsaturated double bond or conjugate structure (Figures 6(a) and 6(b)) [34]. In addition, a lot of hydroxyls both in GO-CNF afforded formation of hydrogen bonds with antibiotics molecules. Secondly, the interconnected 3D networks structure of GO-CNF possessed meso- and macropores (Figure 6(c)), which facilitated the free diffusion of antibiotics and guaranteed mass transport to the 3D crosslinking internal structure, and fully exposing the active sites would enhance the opportunity for antibiotics to contact with the macrostructure of GO-CNF via electrostatic attraction. In summary, the GO-CNF for antibiotics adsorption were through $\pi-\pi$ stacking, hydrogen bonds, and electrostatic attraction.

In consideration of the importance of the recyclability for adsorbents, the $R \%$ of antibiotics on GO-CNF during five sequential cycles of adsorption-desorption was shown in Figure 7. GO-CNF exhibited a potential cycling behavior and lost $1.5-2.5 \%$ with around $2.0 \%$ in five cycles, which had been reduced by $2.5 \%, 1.9 \%, 2.3 \%$, and $1.5 \%$ for DXC, CTC, OTC, and TC, respectively, compared with that in first cycle. In other words, the valve of $R \%$ was $75.0 \%, 79.5 \%$, $72.5 \%$, and $66.1 \%$ for DXC, CTC, OTC, and TC, respectively. These results illustrated that GO-CNF could be still efficiently reused through five regeneration cycles, which could be an efficient, economical, and potential adsorbent for antibiotics removal.

\section{Conclusions}

In conclusion, cellulose nanofibril/graphene oxide hybrid (GO-CNF) aerogel with interconnected 3D network structure had been successfully prepared through one-step ultrasonication method. GO-CNF exhibited superior adsorption performance towards this four kinds of antibiotics (469.7, $396.5,386.5$, and $343.8 \mathrm{mg} \cdot \mathrm{g}^{-1}$ for DXC, CTC, OTC, and TC, resp.), which was attributed to the effects such as chemical reaction ( $\pi-\pi$ stacking and hydrogen bonds) and their pore network microstructure. Furthermore, the simulated equilibrium data exhibited a Langmuir adsorption isotherm and the pseudo-second-order model was well fitted in adsorption kinetic. Importantly, with reusability, as well as ease of separation operation, the GO-CNF might be an efficient and economical adsorbent in antibiotics removal application. 


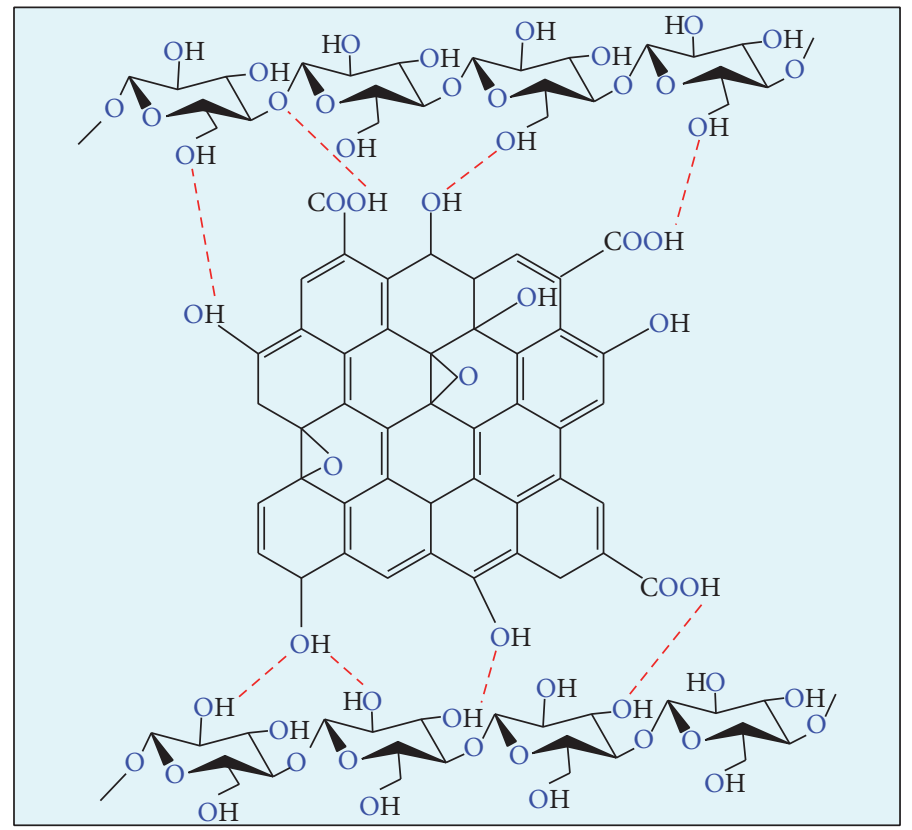

(a)

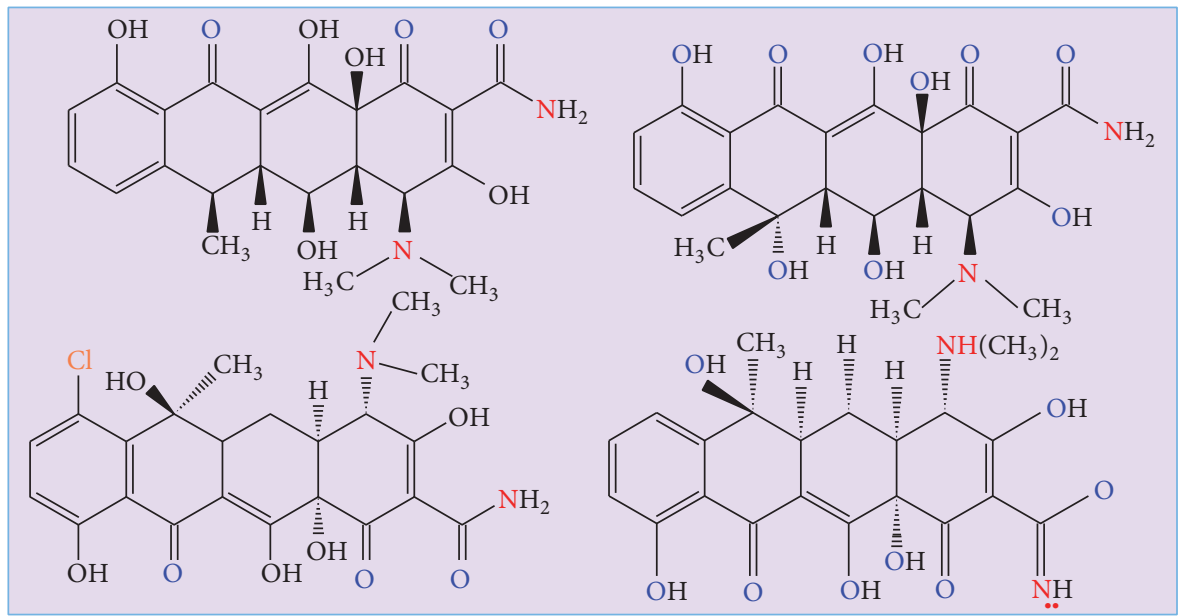

(b)

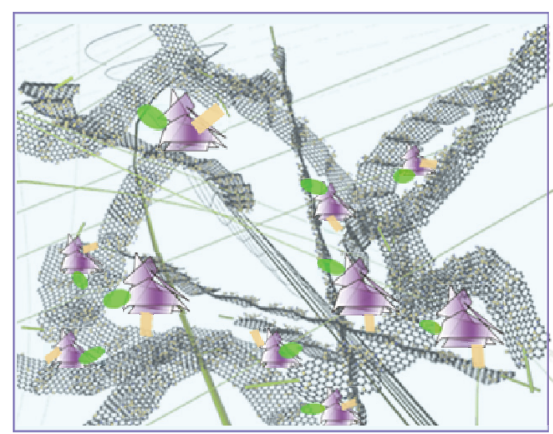

GO-CNF

Antibiotics

$3 \mathrm{D}$ of antibiotics

Hydrogen bonds $3 \mathrm{D}$ of GO-CNF

(c)

FIgURE 6: Schematic diagram of the GO-CNF for antibiotics adsorption. 


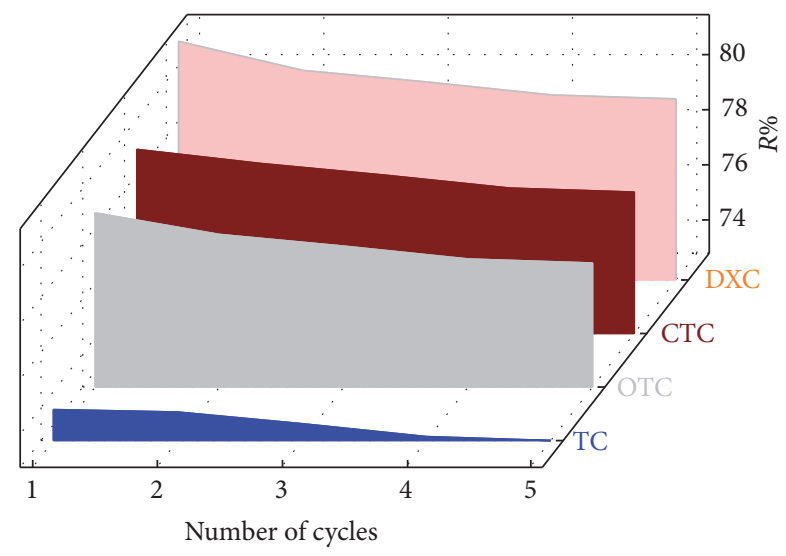

FIGURE 7: Reusability of GO-CNF for antibiotics removal in five cycles.

\section{Conflicts of Interest}

The authors declare that there are no conflicts of interest regarding the publication of this manuscript.

\section{Acknowledgments}

The work was financially supported by Zhejiang Provincial Natural Science Foundation of China (no. LZ14C160001), Public Projects of Zhejiang Province (no. 2015C32014), Scientific Research Foundation of Zhejiang A\&F University (Grant no. 2014FR077), the Fund for Innovative Research Team of Forestry Engineering Discipline (101-206001000713), Cooperation Project of Zhejiang Province and China Forestry Academy (Grant no. 2014SY13), and the Fund for National College students' Innovative Entrepreneurial Training Plan (201610341014).

\section{References}

[1] A. K. Sarmah, M. T. Meyer, and A. B. A. Boxall, "A global perspective on the use, sales, exposure pathways, occurrence, fate and effects of veterinary antibiotics (VAs) in the environment," Chemosphere, vol. 65, no. 5, pp. 725-759, 2006.

[2] X.-S. Miao, F. Bishay, M. Chen, and C. D. Metcalfe, "Occurrence of antimicrobials in the final effluents of wastewater treatment plants in Canada," Environmental Science and Technology, vol. 38, no. 13, pp. 3533-3541, 2004.

[3] A. Alsbaiee, B. J. Smith, L. Xiao, Y. Ling, D. E. Helbling, and W. R. Dichtel, "Rapid removal of organic micropollutants from water by a porous $\beta$-cyclodextrin polymer," Nature, vol. 529, no. 7585, pp. 190-194, 2016.

[4] D. Zhang, J. Yin, J. Zhao, H. Zhu, and C. Wang, "Adsorption and removal of tetracycline from water by petroleum coke-derived highly porous activated carbon," Journal of Environmental Chemical Engineering, vol. 3, no. 3, pp. 1504-1512, 2015.

[5] J. Porras, C. Bedoya, J. Silva-Agredo, A. Santamaría, J. J. Fernández, and R. A. Torres-Palma, "Role of humic substances in the degradation pathways and residual antibacterial activity during the photodecomposition of the antibiotic ciprofloxacin in water," Water Research, vol. 94, pp. 1-9, 2016.
[6] T. Zhou, X. Wu, Y. Zhang, J. Li, and T.-T. Lim, "Synergistic catalytic degradation of antibiotic sulfamethazine in a heterogeneous sonophotolytic goethite/oxalate Fenton-like system," Applied Catalysis B: Environmental, vol. 136-137, pp. 294-301, 2013.

[7] E. A. Serna-Galvis, J. Silva-Agredo, A. L. Giraldo-Aguirre, O. A. Flórez-Acosta, and R. A. Torres-Palma, "High frequency ultrasound as a selective advanced oxidation process to remove penicillinic antibiotics and eliminate its antimicrobial activity from water," Ultrasonics Sonochemistry, vol. 31, pp. 276-283, 2016.

[8] Y. Liu, X. He, Y. Fu, and D. D. Dionysiou, "Degradation kinetics and mechanism of oxytetracycline by hydroxyl radical-based advanced oxidation processes," Chemical Engineering Journal, vol. 284, pp. 1317-1327, 2016.

[9] S. F. Yang, C. F. Lin, C. J. Wu, K. K. Ng, A. Y. Lin, and P. K. Hong, "Fate of sulfonamide antibiotics in contact with activated sludge-sorption and biodegradation," Water Research, vol. 46, no. 4, pp. 1301-1308, 2012.

[10] R. Ocampo-Pérez, J. Rivera-Utrilla, C. Gómez-Pacheco, M. Sánchez-Polo, and J. J. López-Peñalver, "Kinetic study of tetracycline adsorption on sludge-derived adsorbents in aqueous phase," Chemical Engineering Journal, vol. 213, pp. 88-96, 2012.

[11] Y.-L. Zou, H. Huang, M. Chu, J. W. Lin, D.-Q. Yin, and Y.$\mathrm{N}$. Li, "Adsorption research of tetracycline from water by $\mathrm{HCl}$ modified zeolite," Advanced Materials Research, vol. 573-574, pp. 43-47, 2012.

[12] P. Liu, W.-J. Liu, H. Jiang, J.-J. Chen, W.-W. Li, and H.-Q. Yu, "Modification of bio-char derived from fast pyrolysis of biomass and its application in removal of tetracycline from aqueous solution," Bioresource Technology, vol. 121, pp. 235-240, 2012.

[13] K. Li, F. Ji, Y. Liu et al., "Adsorption removal of tetracycline from aqueous solution by anaerobic granular sludge: equilibrium and kinetic studies," Water Science \& Technology, vol. 67, no. 7, pp. 1490-1496, 2013.

[14] D. H. Carrales-Alvarado, R. Ocampo-Pérez, R. Leyva-Ramos, and J. Rivera-Utrilla, "Removal of the antibiotic metronidazole by adsorption on various carbon materials from aqueous phase," Journal of Colloid and Interface Science, vol. 436, pp. 276-285, 2014.

[15] V. Rakić, V. Rac, M. Krmar, O. Otman, and A. Auroux, "The adsorption of pharmaceutically active compounds from aqueous solutions onto activated carbons," Journal of Hazardous Materials, vol. 282, pp. 141-149, 2015.

[16] F. Yu, J. Ma, and S. Han, "Adsorption of tetracycline from aqueous solutions onto multi-walled carbon nanotubes with different oxygen contents," Scientific Reports, vol. 4, article 5326, 2014.

[17] M. C. Ncibi and M. Sillanpää, "Optimized removal of antibiotic drugs from aqueous solutions using single, double and multiwalled carbon nanotubes," Journal of Hazardous Materials, vol. 298, pp. 102-110, 2015.

[18] J. Xu, G.-P. Sheng, Y. Ma, L.-F. Wang, and H.-Q. Yu, "Roles of extracellular polymeric substances (EPS) in the migration and removal of sulfamethazine in activated sludge system," Water Research, vol. 47, no. 14, pp. 5298-5306, 2013.

[19] P. Liao, Z. Zhan, J. Dai et al., "Adsorption of tetracycline and chloramphenicol in aqueous solutions by bamboo charcoal: a batch and fixed-bed column study," Chemical Engineering Journal, vol. 228, pp. 496-505, 2013. 
[20] A. C. Pierre and G. M. Pajonk, "Chemistry of aerogels and their applications," Chemical Reviews, vol. 102, no. 11, pp. 4243-4265, 2002.

[21] G. Zu, J. Shen, W. Wang et al., "Robust, highly thermally stable, core-shell nanostructured metal oxide aerogels as hightemperature thermal superinsulators, adsorbents, and catalysts," Chemistry of Materials, vol. 26, no. 19, pp. 5761-5772, 2014.

[22] E. E. Ghadim, F. Manouchehri, G. Soleimani, H. Hosseini, S. Kimiagar, and S. Nafisi, "Adsorption properties of tetracycline onto graphene oxide: equilibrium, kinetic and thermodynamic studies," PLoS ONE, vol. 8, no. 11, Article ID e79254, 2013.

[23] H. Sun, L. Cao, and L. Lu, "Magnetite/reduced graphene oxide nanocomposites: one step solvothermal synthesis and use as a novel platform for removal of dye pollutants," Nano Research, vol. 4, no. 6, pp. 550-562, 2011.

[24] P. Tan, J. Sun, Y. Hu et al., "Adsorption of $\mathrm{Cu}^{2+}, \mathrm{Cd}^{2+}$ and $\mathrm{Ni}^{2+}$ from aqueous single metal solutions on graphene oxide membranes," Journal of Hazardous Materials, vol. 297, pp. 251260, 2015.

[25] J. Chen, B. Yao, C. Li, and G. Shi, "An improved Hummers method for eco-friendly synthesis of graphene oxide," Carbon, vol. 64, pp. 225-229, 2013.

[26] W. Zhang, Y. Zhang, C. Lu, and Y. Deng, "Aerogels from crosslinked cellulose nano/micro-fibrils and their fast shape recovery property in water," Journal of Materials Chemistry, vol. 22, no. 23, pp. 11642-11650, 2012.

[27] R. T. Olsson, M. A. S. Azizi Samir, G. Salazar-Alvarez et al., "Making flexible magnetic aerogels and stiff magnetic nanopaper using cellulose nanofibrils as templates," Nature Nanotechnology, vol. 5, no. 8, pp. 584-588, 2010.

[28] Z. Fan, K. Wang, T. Wei, J. Yan, L. Song, and B. Shao, "An environmentally friendly and efficient route for the reduction of graphene oxide by aluminum powder," Carbon, vol. 48, no. 5, pp. 1686-1689, 2010.

[29] F. Xiao, S. Yang, Z. Zhang et al., "Scalable synthesis of freestanding sandwich-structured graphene/polyaniline/graphene nanocomposite paper for flexible all-solid-state supercapacitor," Scientific Reports, vol. 5, article 9359, 2015.

[30] K. S. Suslick, "Sonochemistry," Science, vol. 247, no. 4949, pp. 1439-1445, 1990.

[31] H. Sehaqui, Q. Zhou, and L. A. Berglund, "High-porosity aerogels of high specific surface area prepared from nanofibrillated cellulose (NFC)," Composites Science and Technology, vol. 71, no. 13, pp. 1593-1599, 2011.

[32] H. Wang, X. Yuan, Y. Wu et al., "Adsorption characteristics and behaviors of graphene oxide for $\mathrm{Zn}$ (II) removal from aqueous solution," Applied Surface Science, vol. 279, pp. 432-440, 2013.

[33] A. Y. Romanchuk, A. S. Slesarev, S. N. Kalmykov, D. V. Kosynkin, and J. M. Tour, "Graphene oxide for effective radionuclide removal," Physical Chemistry Chemical Physics, vol. 15, no. 7, pp. 2321-2327, 2013.

[34] L.-H. Jiang, Y.-G. Liu, G.-M. Zeng et al., "Removal of $17 \beta$ estradiol by few-layered graphene oxide nanosheets from aqueous solutions: external influence and adsorption mechanism," Chemical Engineering Journal, vol. 284, pp. 93-102, 2016. 

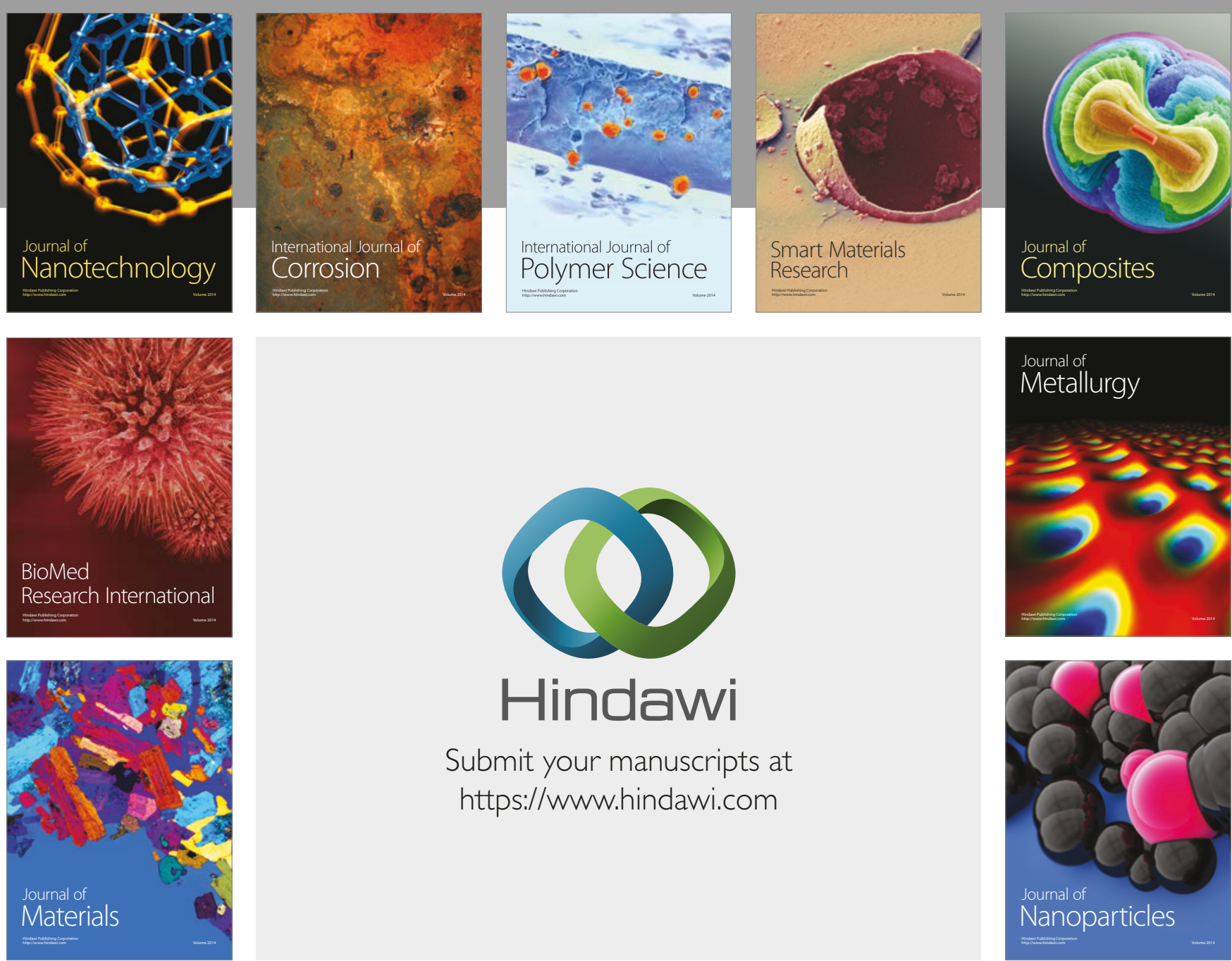

\section{Hindawi}

Submit your manuscripts at

https://www.hindawi.com
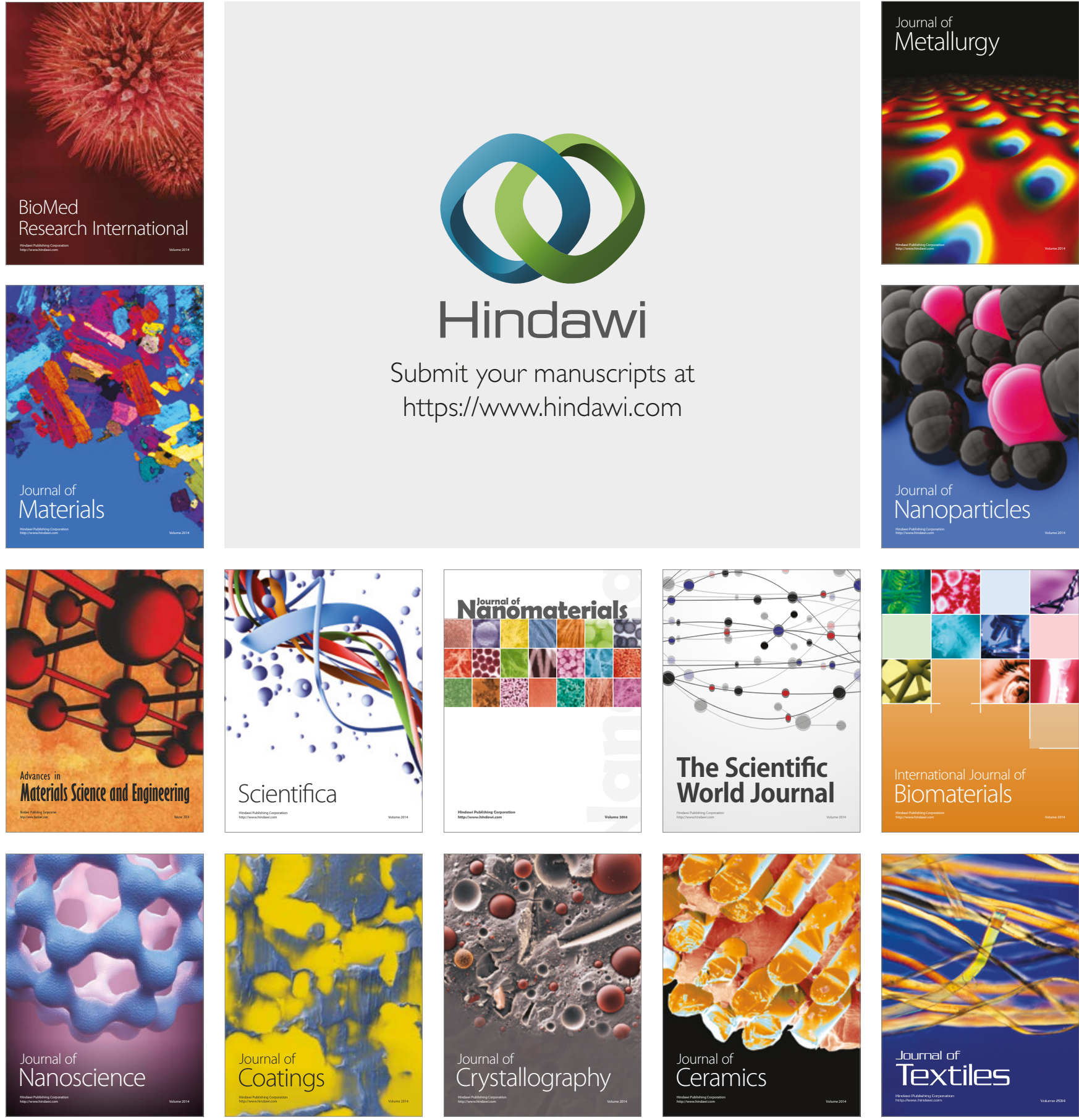

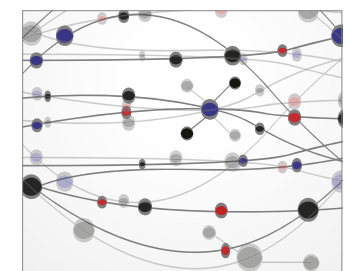

The Scientific World Journal
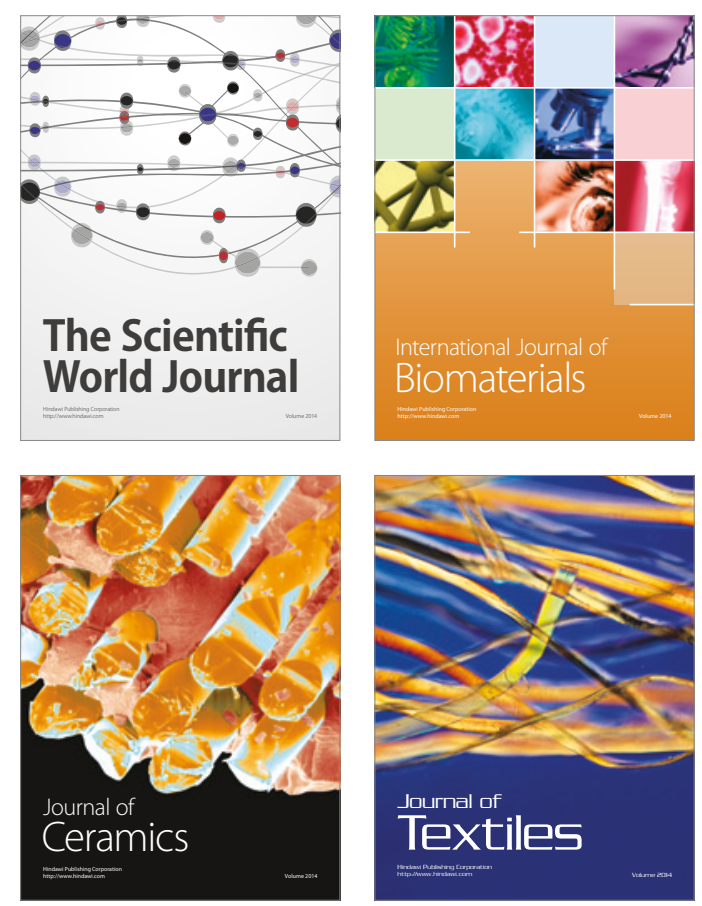